\title{
Probing chiral interactions up to next-to-next-to-next-to-leading order in medium-mass nuclei
}

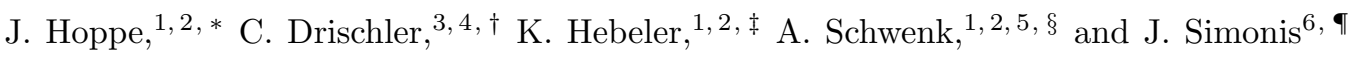 \\ ${ }^{1}$ Institut für Kernphysik, Technische Universität Darmstadt, 64289 Darmstadt, Germany \\ ${ }^{2}$ ExtreMe Matter Institute EMMI, GSI Helmholtzzentrum für Schwerionenforschung GmbH, 64291 Darmstadt, Germany \\ ${ }^{3}$ Department of Physics, University of California, Berkeley, CA 94720 \\ ${ }^{4}$ Lawrence Berkeley National Laboratory, Berkeley, CA 94720 \\ ${ }^{5}$ Max-Planck-Institut für Kernphysik, Saupfercheckweg 1, 69117 Heidelberg, Germany \\ ${ }^{6}$ Institut für Kernphysik and PRISMA Cluster of Excellence, Johannes Gutenberg-Universität, 55099 Mainz, Germany
}

\begin{abstract}
We study ground-state energies and charge radii of closed-shell medium-mass nuclei based on novel chiral nucleon-nucleon $(N N)$ and three-nucleon $(3 N)$ interactions, with a focus on exploring the connections between finite nuclei and nuclear matter. To this end, we perform in-medium similarity renormalization group (IM-SRG) calculations based on chiral interactions at next-to-leading order (NLO), $\mathrm{N}^{2} \mathrm{LO}$, and $\mathrm{N}^{3} \mathrm{LO}$, where the $3 N$ interactions at $\mathrm{N}^{2} \mathrm{LO}$ and $\mathrm{N}^{3} \mathrm{LO}$ are fit to the empirical saturation point of nuclear matter and to the triton binding energy. Our results for energies and radii at $\mathrm{N}^{2} \mathrm{LO}$ and $\mathrm{N}^{3} \mathrm{LO}$ overlap within uncertainties, and the cutoff variation of the interactions is within the EFT uncertainty band. We find underbound ground-state energies, as expected from the comparison to the empirical saturation point. The radii are systematically too large, but the agreement with experiment is better. We further explore variations of the $3 N$ couplings to test their sensitivity in nuclei. While nuclear matter at saturation density is quite sensitive to the $3 N$ couplings, we find a considerably weaker dependence in medium-mass nuclei. In addition, we explore a consistent momentum-space SRG evolution of these $N N$ and $3 N$ interactions, exhibiting improved many-body convergence. For the SRG-evolved interactions, the sensitivity to the $3 N$ couplings is found to be stronger in medium-mass nuclei.
\end{abstract}

\section{INTRODUCTION}

The development of improved nucleon-nucleon $(N N)$ and three-nucleon $(3 N)$ interactions within chiral effective field theory (EFT) for ab initio studies of atomic nuclei and infinite nuclear matter is currently a very active field of research [1 6]. While none of the presently available interactions is able to simultaneously describe experimental ground-state energies and charge radii of nuclei over a wide range of the nuclear chart, recent calculations not unexpectedly indicate a strong correlation between predictions for medium-mass nuclei and nuclear matter properties [1, 7 11]. Although a systematic and quantitative understanding of this correlation is still missing, these studies highlight the significance of realistic saturation properties for the construction of next-generation nuclear forces. In particular, calculations based on interactions fitted only to $N N$ and few-body observables tend to exhibit significant deviations from experiment for heavier nuclei [2, 12, 13]. Remarkably, one exception to this general trend was found in Ref. [7. In this work, a family of $N N$ plus $3 N$ interactions was constructed using similarity renormalization group (SRG) evolved $N N$ interactions combined with the leading chiral $3 N$ forces fitted to the ${ }^{3} \mathrm{H}$ binding energy and the charge radius of

\footnotetext{
* Email: jhoppe@theorie.ikp.physik.tu-darmstadt.de

$\dagger$ Email: cdrischler@berkeley.edu

¥ Email: kai.hebeler@physik.tu-darmstadt.de

$\S$ Email: schwenk@physik.tu-darmstadt.de

I Email: simonis@uni-mainz.de
}

${ }^{4} \mathrm{He}$. While all constructed interactions lead to a reasonable description of the empirical saturation point, the ground-state energies for closed-shell nuclei ranging from ${ }^{4} \mathrm{He}$ to ${ }^{100} \mathrm{Sn}$ are well reproduced for one particular interaction ("1.8/2.0"), whereas charge radii are somewhat too small [10, 14. However, the physical reasons of this remarkable agreement with this specific $N N+3 N$ interaction is still an open question.

These findings suggest incorporating experimental constraints of heavier nuclei directly into the fitting process of nuclear interactions, as was done in Ref. 1. Naturally, results based on these interactions show in general better agreement with experiment for medium-mass nuclei, but $N N$ scattering phase-shifts can only be reproduced to rather low energies, when considering chiral interactions up to next-to-next-to-leading order $\left(\mathrm{N}^{2} \mathrm{LO}\right)$. In Ref. [6], a complementary strategy was pursued to fit the $3 N$ low-energy couplings $c_{D}$ and $c_{E}$ for fixed $N N$ interactions to the ${ }^{3} \mathrm{H}$ binding energy and the saturation region of nuclear matter using a novel many-body perturbation theory framework for nuclear matter. It was found that, based on the $N N$ interactions of Ref. [5], a reasonable reproduction of the saturation point can be obtained for all interactions at $\mathrm{N}^{2} \mathrm{LO}$ and $\mathrm{N}^{3} \mathrm{LO}$ and for different cutoffs $\Lambda=450$ and $500 \mathrm{MeV}$. In this work, we extend this study to interactions with a lower cutoff $\Lambda=420 \mathrm{MeV}$ and investigate in detail the properties of medium-mass nuclei based on these interactions using the $a b$ initio in-medium similarity renormalization group (IM-SRG). This work thus presents the first $\mathrm{N}^{3} \mathrm{LO}$ calculations of medium-mass nuclei.

The paper is organized as follows: We briefly discuss 
the employed chiral $N N$ plus $3 N$ interactions in Sec. II and the IM-SRG many-body calculations in Sec. III. In Sec. [V] we study the model-space convergence and present results for ground-state energies and charge radii of medium-mass nuclei up to nickel isotopes. We study the effects of consistent momentum-space SRG evolutions of the $N N$ plus $3 N$ interactions and investigate the systematics of the results for nuclei and matter with respect to variations of the low-energy couplings. Finally, we conclude and give an outlook in Sec. $\mathrm{V}$

\section{CHIRAL INTERACTIONS}

As in the recent $\mathrm{N}^{3} \mathrm{LO}$ nuclear matter study [6], we use the nonlocal chiral $N N$ interactions of Entem, Machleidt, and Nosyk (EMN) [5] with cutoffs $\Lambda=450$ and $500 \mathrm{MeV}$. In addition, we consider a softer $\mathrm{N}^{3} \mathrm{LO}$ interaction with cutoff $\Lambda=420 \mathrm{MeV}$ [15]. The $3 N$ interactions are regularized via a nonlocal regulator

$$
f_{\Lambda_{3 N}}(p, q)=\exp \left[-\left(\frac{4 p^{2}+3 q^{2}}{4 \Lambda_{3 N}^{2}}\right)^{4}\right],
$$

where $p$ and $q$ are the magnitudes of the relative momenta $\mathbf{p}$ and $\mathbf{q}$, respectively [16, and (by choice) $\Lambda_{3 N}=\Lambda$. In Ref. 6], we studied saturation properties of symmetric nuclear matter of these $N N$ potentials combined with consistent $3 \mathrm{~N}$ forces up to $\mathrm{N}^{3} \mathrm{LO}$ using a new Monte Carlo framework that enables high-order calculations in many-body perturbation theory (MBPT). It was shown that fits to the triton binding energy and the empirical saturation point lead to narrow ranges for the two $3 N$ low-energy couplings $c_{D}$ and $c_{E}$ (note that the fit to nuclear matter was not optimized to high accuracy). The resulting values of the $3 N$ couplings are given in the last row of Table I] In addition to Ref. [6], we explore the softer $\mathrm{N}^{3} \mathrm{LO}$ interaction with $\Lambda=420 \mathrm{MeV}$ for nuclear matter saturation in Fig. 1. For this study, we take $c_{D}=4.0$ (varied in steps of 1 ), to explore the reproduction of the saturation density.

TABLE I. Three-nucleon couplings $c_{D}$ and $c_{E}$ for the $\mathrm{N}^{3} \mathrm{LO}$ EMN $N N$ potentials 420,450 , and $500 \mathrm{MeV}$ [5, 15, which reproduce the experimental ${ }^{3} \mathrm{H}$ ground-state energy $E\left({ }^{3} \mathrm{H}\right)=$ $-8.482 \mathrm{MeV}$ at $\mathrm{N}^{3} \mathrm{LO}$. The last row gives the results from simultaneous fits to the ${ }^{3} \mathrm{H}$ binding energy and the empirical saturation region of symmetric nuclear matter (see Ref. 6] for details for 450 and $500 \mathrm{MeV}$ ). The fits to the empirical saturation point for $\Lambda=420 \mathrm{MeV}$ are shown in Fig. 1 .

\begin{tabular}{cc|cc|cc}
\hline \hline \multicolumn{2}{c|}{$420 \mathrm{MeV}$} & \multicolumn{2}{|c|}{$450 \mathrm{MeV}$} & \multicolumn{2}{c}{$500 \mathrm{MeV}$} \\
$c_{D}$ & $c_{E}$ & $c_{D}$ & $c_{E}$ & $c_{D}$ & $c_{E}$ \\
\hline-5.0 & -2.509 & -5.0 & -2.149 & -5.0 & -2.534 \\
0.0 & -1.558 & 0.0 & -1.321 & 0.0 & -1.848 \\
5.0 & -0.685 & 5.0 & -0.636 & 5.0 & -1.573 \\
\hline 4.0 & -0.853 & 0.25 & -1.280 & -2.75 & -2.190 \\
\hline \hline
\end{tabular}

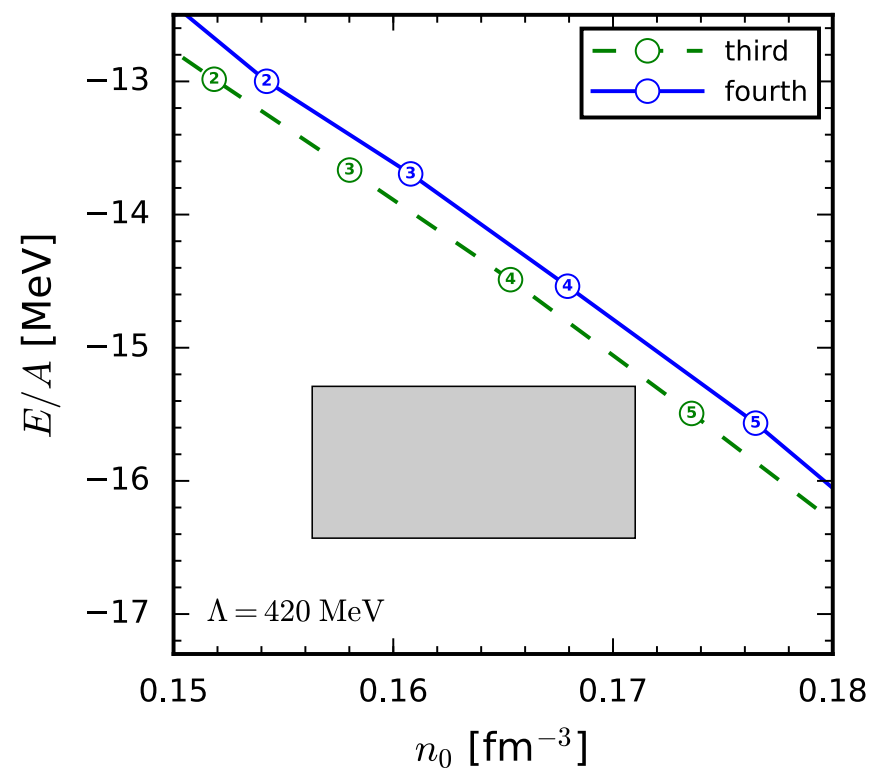

FIG. 1. Saturation point of symmetric nuclear matter at third (green-dashed line) and fourth (blue-solid line) order in MBPT as trajectory of $c_{D}$ (see annotated values inside the circles), while $c_{E}$ is determined by the ${ }^{3} \mathrm{H}$ binding energy. The results are based on the $\mathrm{N}^{3} \mathrm{LO}$ EMN $420 \mathrm{MeV} N N$ potential with consistent $3 N$ forces at $\mathrm{N}^{3} \mathrm{LO}$. For details on the fits to the empirical saturation region (gray box) see Ref. [6].

In this work, we study the properties of medium-mass nuclei for the first time to $\mathrm{N}^{3} \mathrm{LO}$ using the IM-SRG framework. The relative $3 \mathrm{~N}$ matrix elements up to $\mathrm{N}^{3} \mathrm{LO}$ have been calculated in Ref. [17. We consider both unevolved and for the first time consistently momentumspace SRG-evolved $N N$ and $3 N$ interactions following Ref. [18. The SRG can significantly improve the rate of convergence of many-body calculations at the cost of induced many-body forces that may be sizable depending on the resolution scale of interest. Such induced contributions cannot be included beyond the $3 N$ level at the moment, but the residual sensitivity of our results on the flow parameter serves as an estimate of the uncertainty due to neglected higher-body contributions.

\section{IN-MEDIUM SIMILARITY RENORMALIZATION GROUP}

The IM-SRG takes advantage of normal ordering with respect to a chosen reference state and decouples particlehole excitations from the ground state by a continuous sequence of unitary transformations to solve the manybody problem [10, 19,22. Similar to the free-space SRG [23, 24], the flow equation for the Hamiltonian is given by

$$
\frac{\mathrm{d} H(s)}{\mathrm{d} s}=[\eta(s), H(s)]
$$


with the flow parameter $s$ and the generator $\eta(s)$. For the IM-SRG, we take the arctan generator following the work of White [25].

The commutator relation (2) induces up to $A$-body contributions in an $A$-body system. Including all of these induced terms is not feasible at the moment, making a truncation scheme necessary. We use the IM-SRG(2), in which all operators are truncated at the normal-ordered two-body level and apply the Magnus formalism [26, 27. to the flow equations instead of an ordinary differential equation solver. By calculating the unitary transformation underlying the IM-SRG directly, this approach is less memory demanding and faster, especially for operators other than the Hamiltonian.

To calculate charge radii, we also evolve the intrinsic point-proton mean-square radius operator

$$
R_{p}^{2}=\frac{1}{Z} \sum_{i=1}^{A} \frac{1+\tau_{3}^{(i)}}{2}\left(\mathbf{r}_{i}-\mathbf{R}\right)^{2},
$$

using the Magnus formalism, where $\mathbf{r}_{i}(\mathbf{R})$ is the nucleon (nucleus center-of-mass) coordinate. $A$ and $Z$ are the mass and proton number, respectively, and the operator $\left(1+\tau_{3}^{(i)}\right) / 2$ with the third component of the isospin operator $\tau_{3}^{(i)}$, projects on protons. Taking into account the proton and neutron mean-square charge radius $\left\langle r_{p}^{2}\right\rangle=0.770 \mathrm{fm}^{2}$ and $\left\langle r_{n}^{2}\right\rangle=-0.1149 \mathrm{fm}^{2}$ [28, as well as the relativistic Darwin-Foldy correction [29, 30 $3 /\left(4 m_{p}^{2} c^{4}\right)=0.033 \mathrm{fm}^{2}$, and the spin-orbit correction $\left\langle r^{2}\right\rangle_{\text {so }}$ [31, we obtain the mean-square charge radius from

$$
R_{\mathrm{ch}}^{2}=R_{p}^{2}+\left\langle r_{p}^{2}\right\rangle+\frac{N}{Z}\left\langle r_{n}^{2}\right\rangle+\frac{3}{4 m_{p}^{2} c^{4}}+\left\langle r^{2}\right\rangle_{\mathrm{so}},
$$

with neutron number $N$. For further details on the evaluation, we refer to the calculation of charge radii in the IM-SRG in Ref. 10.

\section{RESULTS}

We first study the model-space convergence with respect to the harmonic-oscillator single-particle basis with quantum numbers $e=2 n+l \leqslant e_{\mathrm{Max}}$ and oscillator frequency $\hbar \omega$. As usual, an additional cut for the $3 N$ interaction matrix elements in the single-particle basis is introduced by $e_{1}+e_{2}+e_{3} \leqslant E_{3 \mathrm{Max}}<3 e_{\mathrm{Max}}$. For the single-particle $3 N$ matrix elements, our results are based on Ref. [10]. For the transformation of relative $3 N$ matrix elements to the single-particle basis, we apply the truncation $\mathcal{J} \leqslant \mathcal{J}_{\text {Max }}=9 / 2$ in the relative total three-body angular momentum $\mathcal{J}$ for unevolved interactions with the relative total two-body angular momentum $J_{\max }=8,7,6$ for $\mathcal{J} \leqslant 5 / 2, \mathcal{J}=7 / 2$, and $\mathcal{J}=9 / 2$, respectively. For SRG-evolved interactions we use $\mathcal{J}_{\max }=7 / 2$ and $J_{\max }=5$. Contributions to the ground-state energies beyond these limits for these interactions are expected to be at the level of $\mathrm{MeV}$, which is small compared to the interaction sensitivity explored in the following.

In Figs. 2 and 3 we show the ground-state energies and charge radii of ${ }^{16} \mathrm{O}$ and ${ }^{56} \mathrm{Ni}$ for the $N N$-only and $N N$ plus $3 N$ interactions as a function of the harmonicoscillator frequency $\hbar \omega$ and for different model-space truncations. While we observe converged results at $\hbar \omega$ $\approx 20-24 \mathrm{MeV}$ for ${ }^{16} \mathrm{O}$ for $N N+3 N$ interactions, the results for ${ }^{56} \mathrm{Ni}$ are fully converged only with respect to the single-particle basis $e_{\mathrm{Max}}$. Increasing the $3 N$ cut $E_{3 \mathrm{Max}}$ from 14 to 16 still results in slight changes for energies and radii. Moreover, selecting the optimal frequency for extracting the charge radius of ${ }^{56} \mathrm{Ni}$ is not as clear as for ${ }^{16} \mathrm{O}$, as the results show an unusual convergence behavior with $\hbar \omega$ and the model-space truncation, which could be due to the $3 N$ cut $E_{3 \mathrm{Max}}$.

For all following results, we choose the frequency for extracting radii consistent with the ground-state energy, keeping in mind that the results for the charge radii of the nickel and heavier calcium isotopes are somewhat less converged. Calculations based on $N N$-only interactions are well converged for both nuclei and the optimal $\hbar \omega$ is shifted to slightly larger values. Generally, we find that $3 N$ interactions have a significant impact on the ground-state energies and charge radii. In both nuclei, ${ }^{16} \mathrm{O}$ and ${ }^{56} \mathrm{Ni}, 3 N$ interactions provide repulsive contributions, leading to significantly reduced binding energies and increased charge radii. Compared to experimental values, we find an underbinding of about $30 \mathrm{MeV}$ $(200 \mathrm{MeV})$ for ${ }^{16} \mathrm{O}\left({ }^{56} \mathrm{Ni}\right)$, whereas the charge radius of ${ }^{16} \mathrm{O}$ turns out to be too large by about $0.2 \mathrm{fm}$.

In the following, we study ${ }^{40} \mathrm{Ca}$ based on consistentlyevolved $N N+3 N$ interactions following Ref. [18. We distinguish three cases: " $N N$-only" (no $3 N$ contributions at all), " $N N+3 N$-induced" ( $N N$ plus induced $3 N$ contributions from the $N N$ SRG evolution in momentum space), and " $N N+3 N$-full" (including also initial $3 N$ interactions in the SRG evolution). Our results for the ground-state energy and charge radius of ${ }^{40} \mathrm{Ca}$ are shown in Figs. 4 and 5 for the $\mathrm{N}^{3} \mathrm{LO}$ EMN $450 \mathrm{MeV}$ and $500 \mathrm{MeV}$ interactions. Note that the radius operator is not free-space SRG evolved.

As in Figs. 2 and 3 , we find significantly decreased binding energies and increased charge radii due to $3 \mathrm{~N}$ interactions, also for the $500 \mathrm{MeV}$ interaction. The " $N N$ only" results exhibit a sizable resolution scale dependence, whereas " $N N+3 N$-induced" leads to very similar results in the studied range $\lambda=1.8-2.2 \mathrm{fm}^{-1}$. This indicates that contributions from neglected induced higherbody interactions are rather insignificant. However, although the results with unevolved interactions appear to be converged with respect to the model space $e_{\text {Max }}$ for $\Lambda=450 \mathrm{MeV}$, they differ significantly from the results with evolved interactions. This indicates that contributions beyond the IM-SRG(2) are indeed relevant. The " $N N+3 N$-full" ground-state energies are remarkably similar for both $\mathrm{N}^{3} \mathrm{LO}$ EMN $450 \mathrm{MeV}$ and $500 \mathrm{MeV}$ interactions. This is most likely due to fitting the $3 N$ 


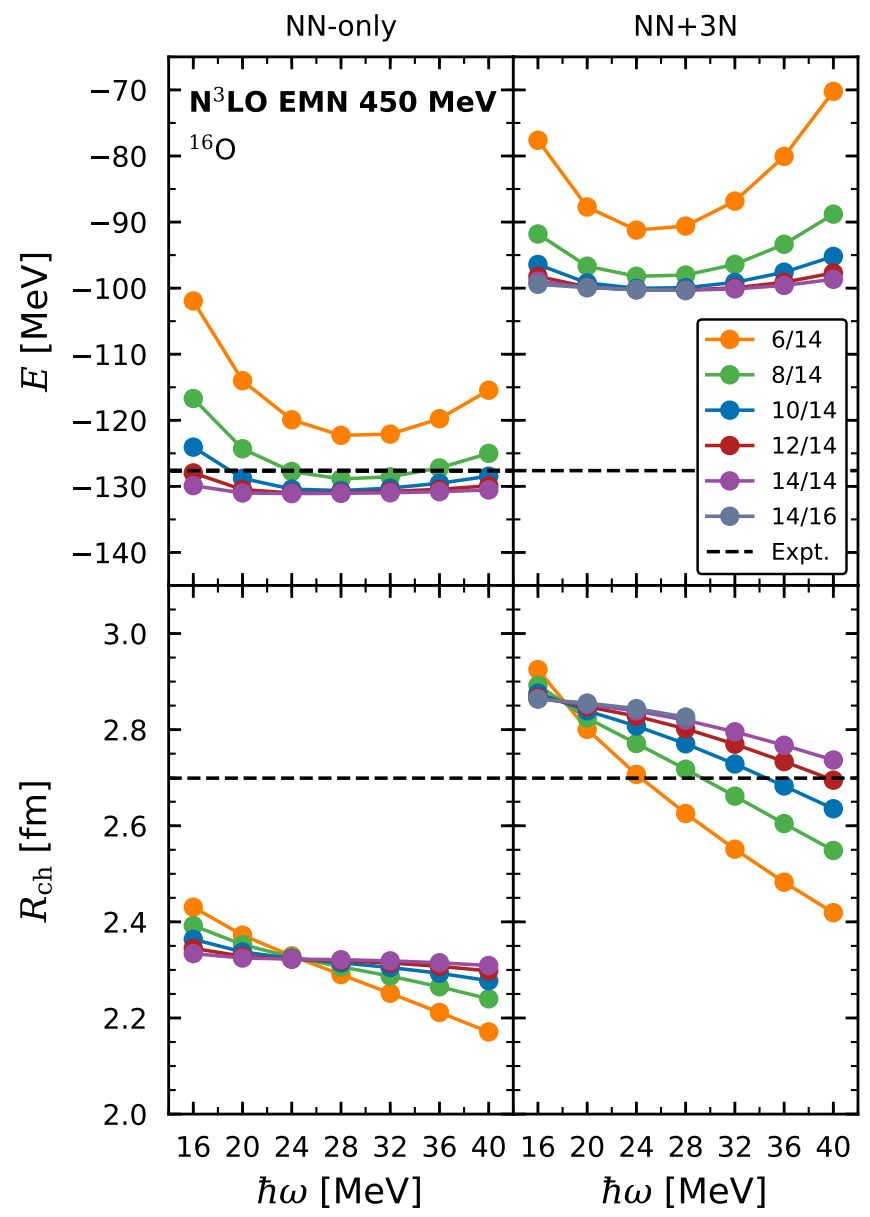

FIG. 2. Ground-state energy (top) and charge radius (bottom panel) of ${ }^{16} \mathrm{O}$ as a function of the harmonic-oscillator frequency $\hbar \omega$ at $\mathrm{N}^{3} \mathrm{LO}$ for the $N N$-only EMN $450 \mathrm{MeV}$ potential and the consistent $N N+3 N$ interaction in the left and right panels, respectively. Results are shown for different sizes of the single-particle basis, $e_{\mathrm{Max}}=6,8,10,12$, and 14 , and for $E_{3 \mathrm{Max}}=14$ and 16 , denoted by $e_{\mathrm{Max}} / E_{3 \mathrm{Max}}$. The experimental values (black-dashed lines) are taken from Refs. [32, 33.

couplings to the same nuclear matter observables. However, as in Figs. 22 and 3, there are similar deficiencies with respect to experiment, with a difference of about $2 \mathrm{MeV}$ per nucleon (see also Fig. 6).

We find similar trends for the charge radius in Fig. 5 . In this case, the " $N N+3 N$-induced" results are very similar for the unevolved interaction and all resolution scales studied, indicating that contributions beyond the IMSRG(2) may be less relevant for this observable. This observation could point to the missing IM-SRG(3) contributions being of short-range character, given that the radius operator is mainly sensitive to long-range contributions. We find again remarkably similar results for both $\mathrm{N}^{3} \mathrm{LO}$ EMN $450 \mathrm{MeV}$ and $500 \mathrm{MeV}$ interactions. In contrast to the results for the ground-state energy (see Fig. (4), we find a better agreement with the experimental charge radius for all " $N N+3 N$-full" calculations (note the scale in Fig. 4 compared to Fig. 5).

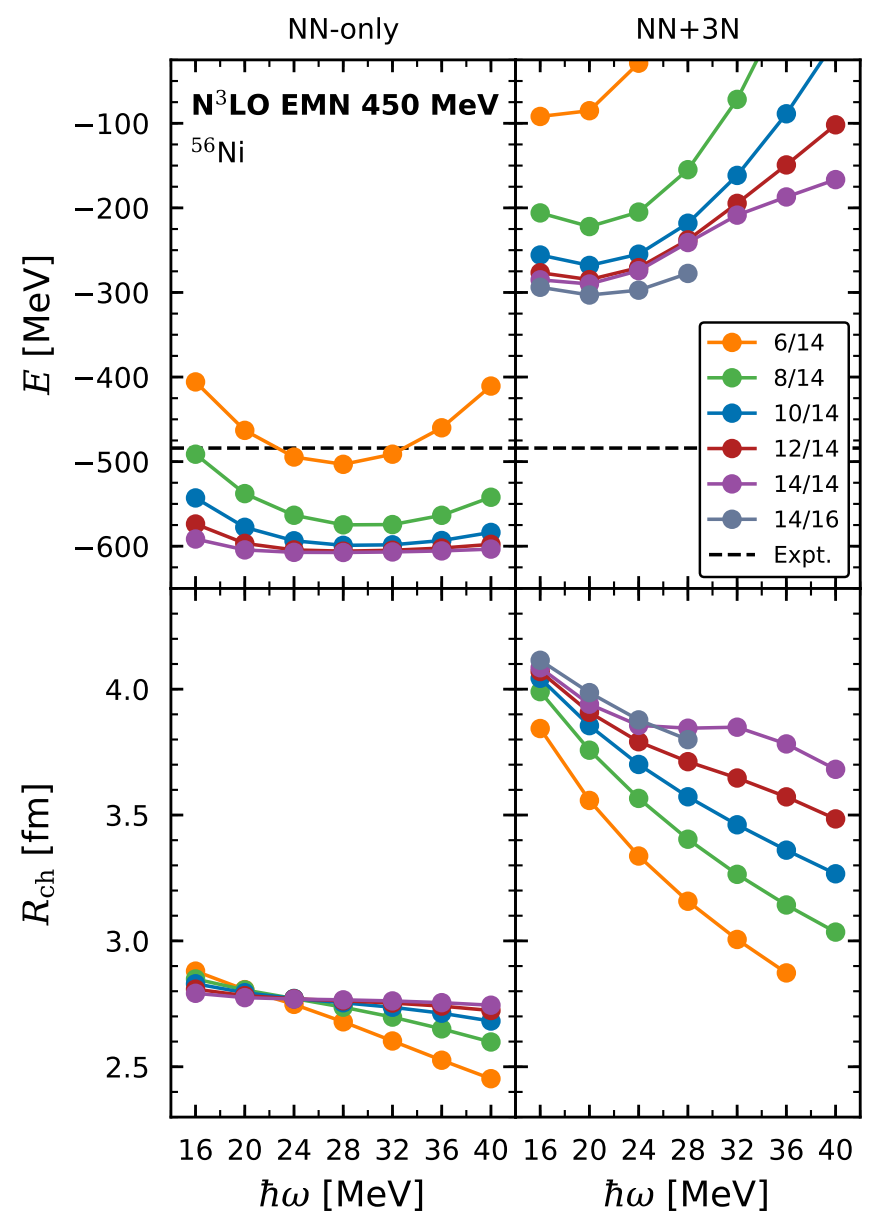

FIG. 3. Same as Fig. 2 but for ${ }^{56} \mathrm{Ni}$.

In the following, we consider a model space of $e_{\operatorname{Max}} / E_{3 \operatorname{Max}}=14 / 14$ and $\hbar \omega=20 \mathrm{MeV}$. In Figs. 6 and 7 , we show results for ground-state energies and charge radii of selected closed-shell oxygen, calcium, and nickel isotopes for the (unevolved) $\mathrm{N}^{3} \mathrm{LO}$ EMN 420, 450, and $500 \mathrm{MeV}$ interactions. In addition, we present results at $\mathrm{NLO}$ and $\mathrm{N}^{2} \mathrm{LO}$ for the EMN $450 \mathrm{MeV}$ interaction. This enables us to provide uncertainty estimates for the order-by-order convergence of the chiral expansion (see, e.g., Ref. [35]). For the orders $i \geqslant 3$ (i.e., $\geqslant \mathrm{N}^{2} \mathrm{LO}$ ), the uncertainty $\Delta X^{(i)}$ for a fixed cutoff is estimated by

$$
\Delta X^{(i)}=\max _{3 \leqslant j \leqslant i}\left(Q^{i+1-j}\left|X^{(j)}-X^{(j-1)}\right|\right),
$$

where $X^{(j)}$ denotes the obtained result at order $j$ in chiral EFT and $Q=m_{\pi} / \Lambda_{b}$ is the ratio of a typical momentum scale over the breakdown scale, with the pion mass $m_{\pi}=140 \mathrm{MeV}$ and we take for the breakdown scale $\Lambda_{b}=500 \mathrm{MeV}$. In Figs. 6 and 7, the uncertainty estimates for the EMN $450 \mathrm{MeV}$ interaction at $\mathrm{N}^{2} \mathrm{LO}$ and $\mathrm{N}^{3} \mathrm{LO}$ are depicted by the blue and orange band, respectively. We have assessed the convergence with respect to $E_{3 \mathrm{Max}}$ by increasing its value to 16 in selected cases, leading to changes of ground-state energies up to about 


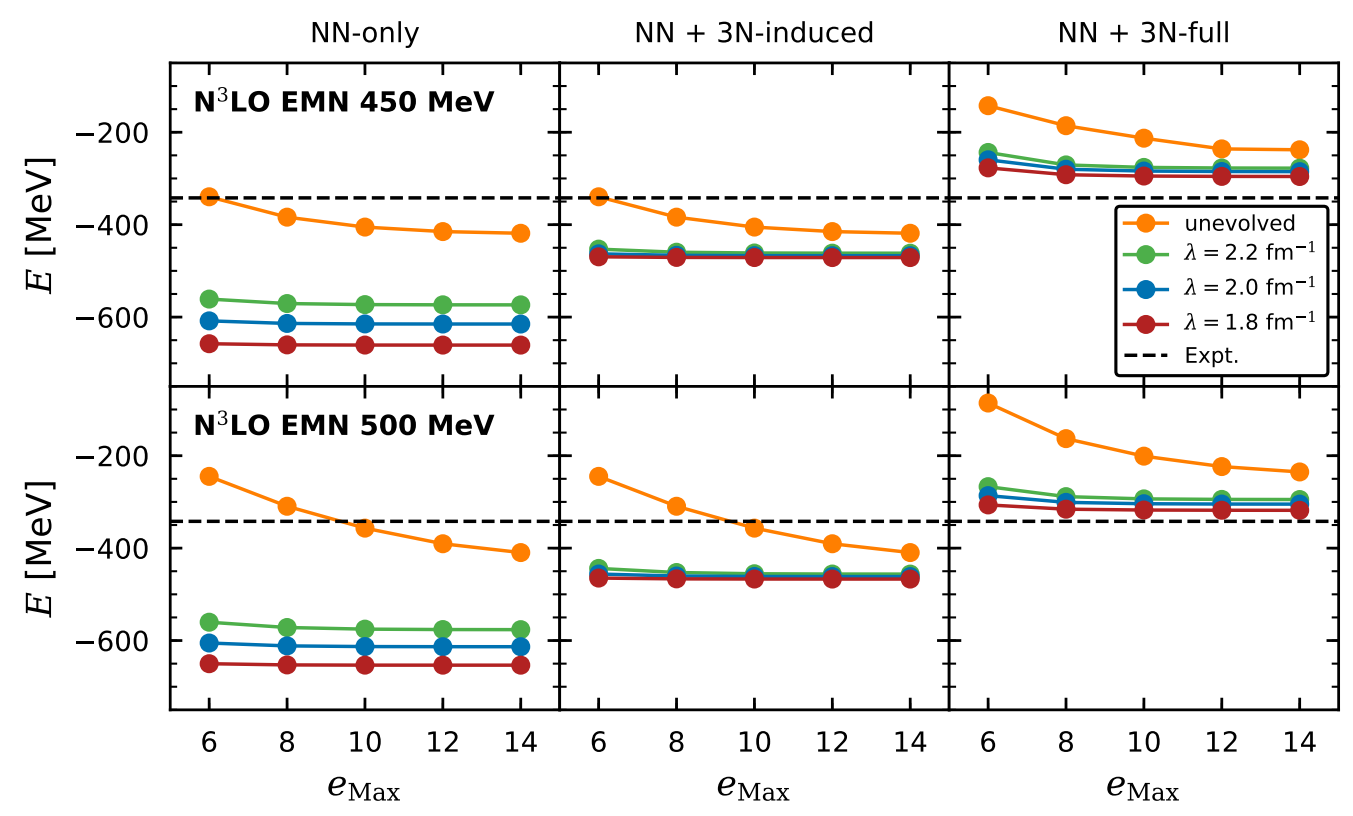

FIG. 4. Ground-state energy of ${ }^{40} \mathrm{Ca}$ as a function of $e_{\mathrm{Max}}$ for the $N N$-only (left), $N N+3 N$-induced (middle), and $N N+3 N$ full (right) interactions of the $\mathrm{N}^{3} \mathrm{LO}$ EMN $450 \mathrm{MeV}$ and $500 \mathrm{MeV}$ potentials, unevolved and SRG-evolved to resolution scales $\lambda=2.2,2.0$, and $1.8 \mathrm{fm}^{-1}$, respectively. Results are shown for $\hbar \omega=20 \mathrm{MeV}$ and $E_{3 \mathrm{Max}}=14$. The experimental value from Ref. [32] is given by the black-dashed line.

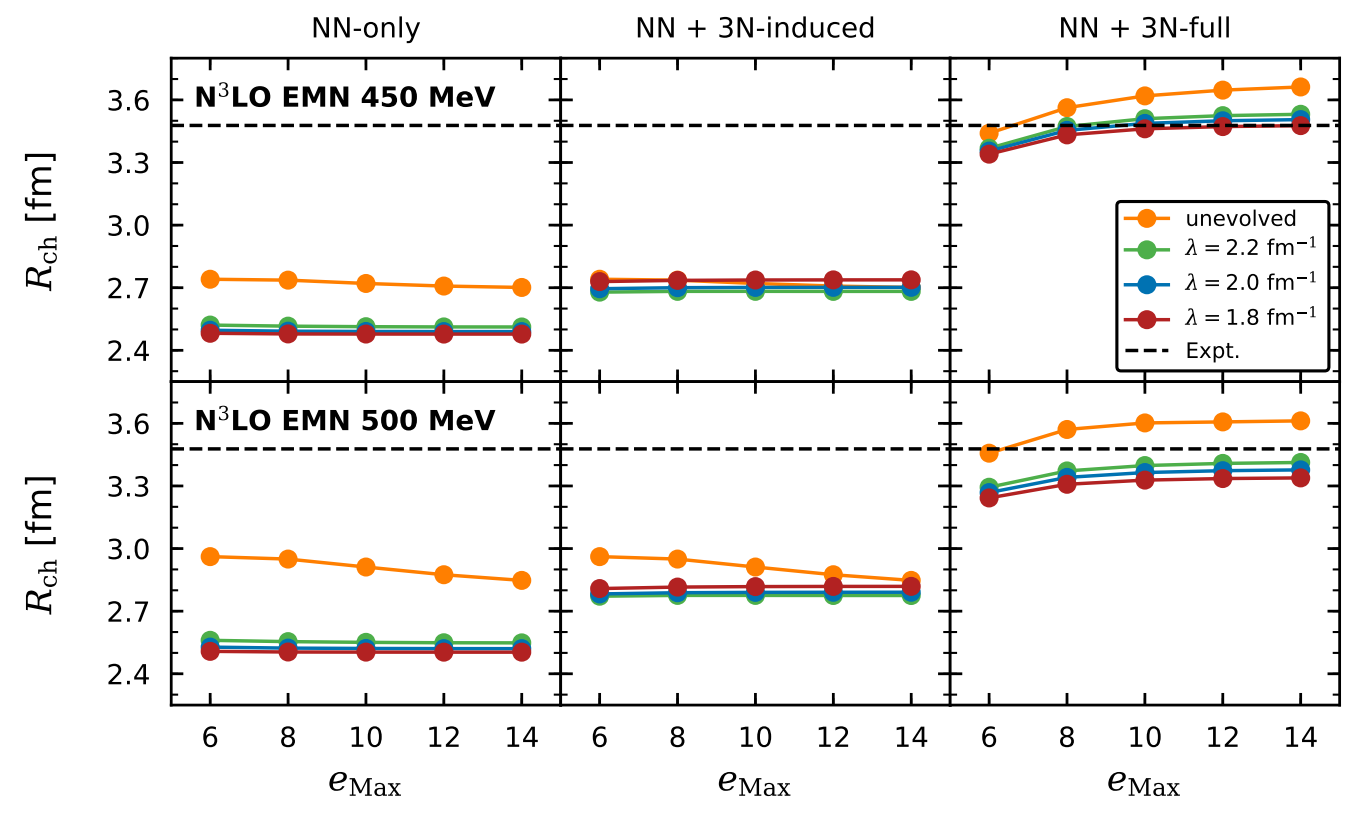

FIG. 5. Same as Fig. 4 but for the charge radius of ${ }^{40} \mathrm{Ca}$. The experimental value is taken from Ref. 33. Note that the results for unevolved interactions are barely visible in some panels, as they are on top of the corresponding evolved results.

$0.1 \mathrm{MeV} / A$ until ${ }^{40} \mathrm{Ca}$ and up to $0.5 \mathrm{MeV} / A$ for ${ }^{68} \mathrm{Ni}$. The changes of radii are only minor. However, as discussed above we note that contributions beyond the IMSRG(2) may be important and need to also be explored explicitly in the future. For comparison, we also include in Figs. 6 and 7 results based on a recently developed $\Delta$-full interaction [1] at $\mathrm{N}^{2} \mathrm{LO}$, using the same model space but $\hbar \omega=16 \mathrm{MeV}$. This also shows the excellent comparison of our IM-SRG(2) calculations with the coupled cluster (CC) results from Ref. [11].

The NLO interaction significantly overbinds all nuclei. Adding $\mathrm{N}^{2} \mathrm{LO}$ leads to substantial repulsive contributions, resulting in an underbinding compared to experiment. The impact of $\mathrm{N}^{3} \mathrm{LO}$ on the ground-state energies is rather small. Overall the results exhibit a systematic order-by-order convergence with overlapping $\mathrm{N}^{2} \mathrm{LO}$ and 


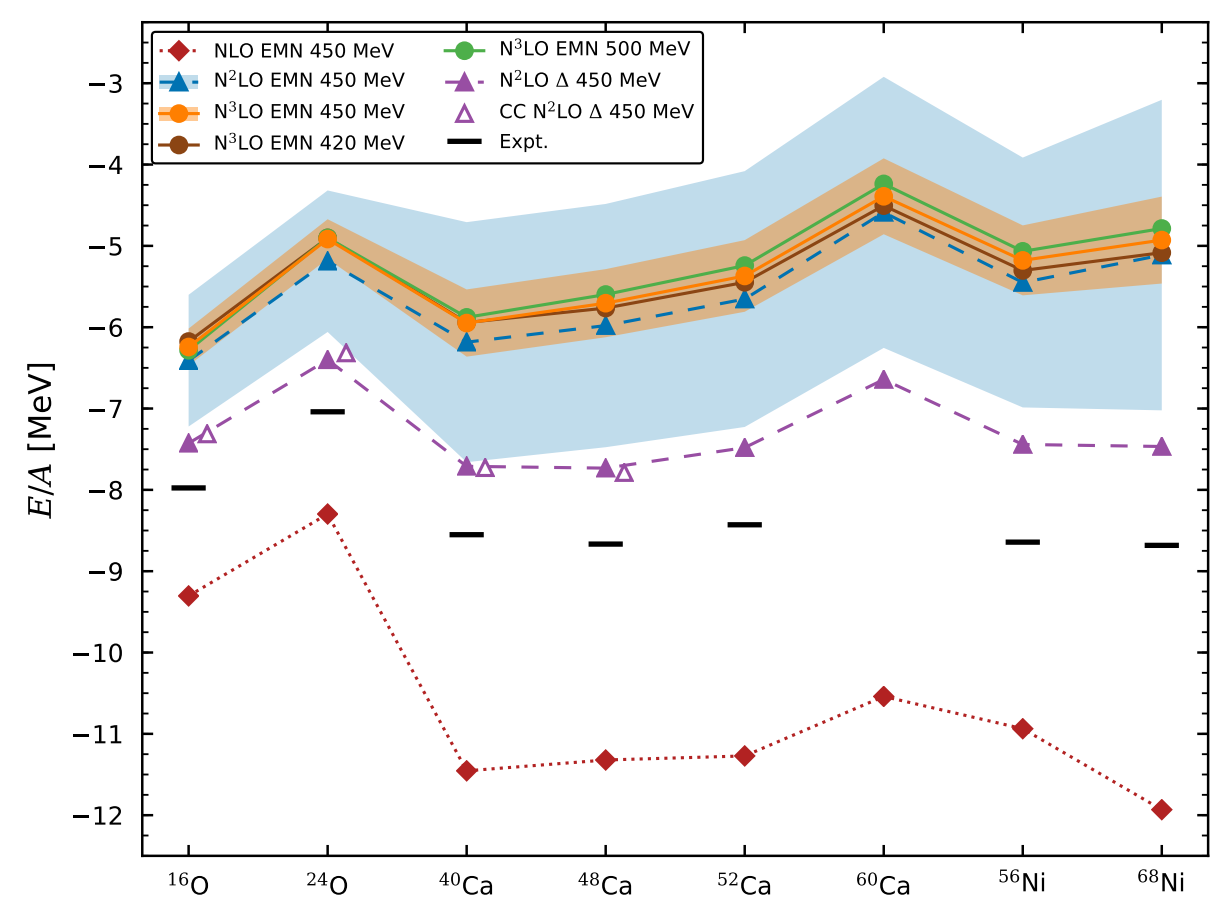

FIG. 6. Ground-state energies per nucleon of selected closed-shell oxygen, calcium, and nickel isotopes. Results are shown at $\mathrm{N}^{3} \mathrm{LO}$ for the EMN potential with cutoffs $\Lambda=420,450$, and $500 \mathrm{MeV}$ depicted by the brown, orange, and green-solid lines and circles, respectively. The $\mathrm{N}^{2} \mathrm{LO}$ results are given by the dashed lines for the EMN $450 \mathrm{MeV}$ potential (blue line and solid up triangles) and the $\Delta$-full interaction (purple line and solid up triangles), while NLO results are displayed by the red-dotted line and diamonds. The open triangles give the coupled cluster (CC) results for the $\Delta$-full interaction from Ref. 11 for comparison. The blue and orange bands give the $\mathrm{N}^{2} \mathrm{LO}$ and $\mathrm{N}^{3} \mathrm{LO}$ uncertainty estimate, respectively, for the EMN $450 \mathrm{MeV}$ interaction. We note that the uncertainty due to the $E_{3 \mathrm{Max}}$ cut is $\lesssim 0.1 \mathrm{MeV} / A$ through ${ }^{40} \mathrm{Ca}$ and increases up to $\sim 0.5 \mathrm{MeV} / A$ for ${ }^{68} \mathrm{Ni}$. Experimental values are taken from Ref. 32.

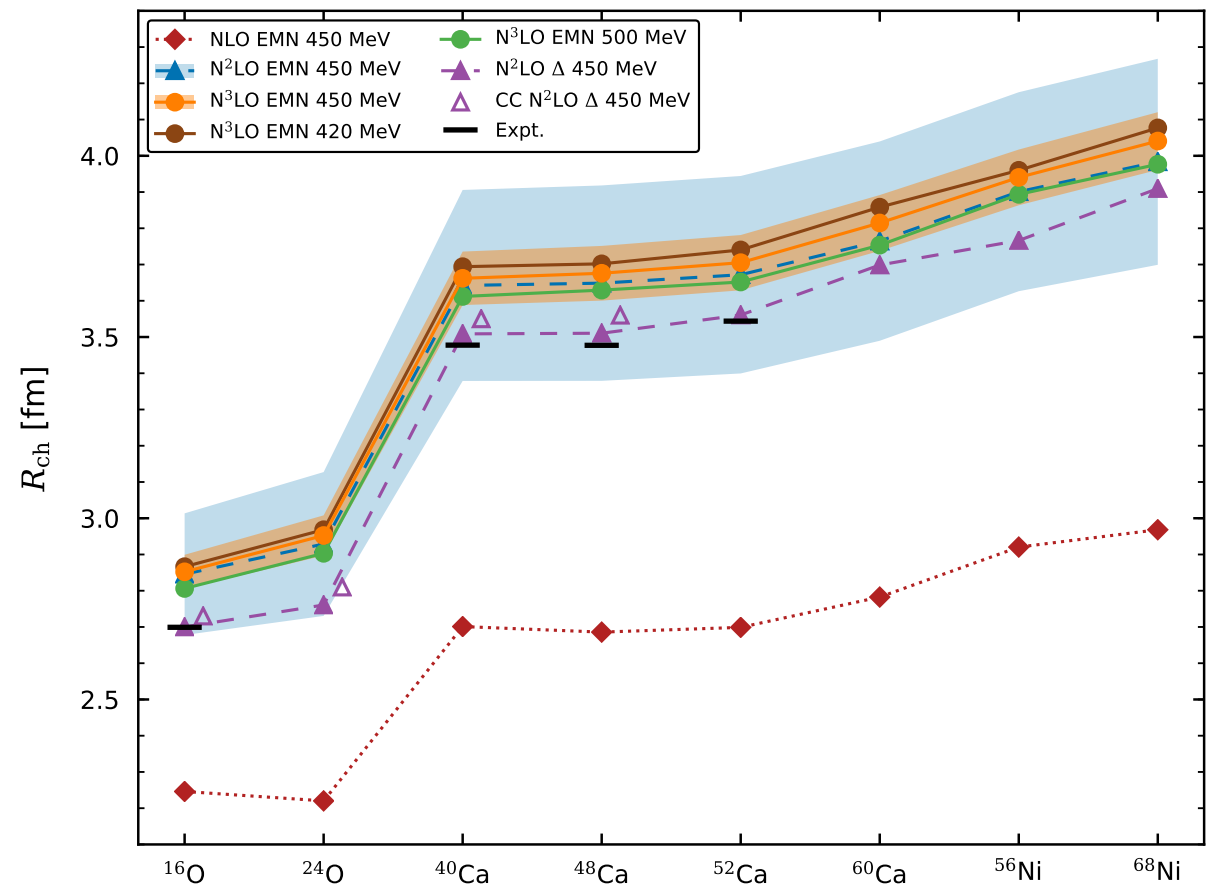

FIG. 7. Same as Fig. 6 but for charge radii. Experimental values are taken from Refs. 33, 34]. Note that the results for the heavier calcium isotopes and beyond are somewhat less converged in $\hbar \omega$ (see text for details).

$\mathrm{N}^{3} \mathrm{LO}$ bands. Moreover, we observe only a weak cutoff dependence at $\mathrm{N}^{3} \mathrm{LO}$, which slightly increases for larger 


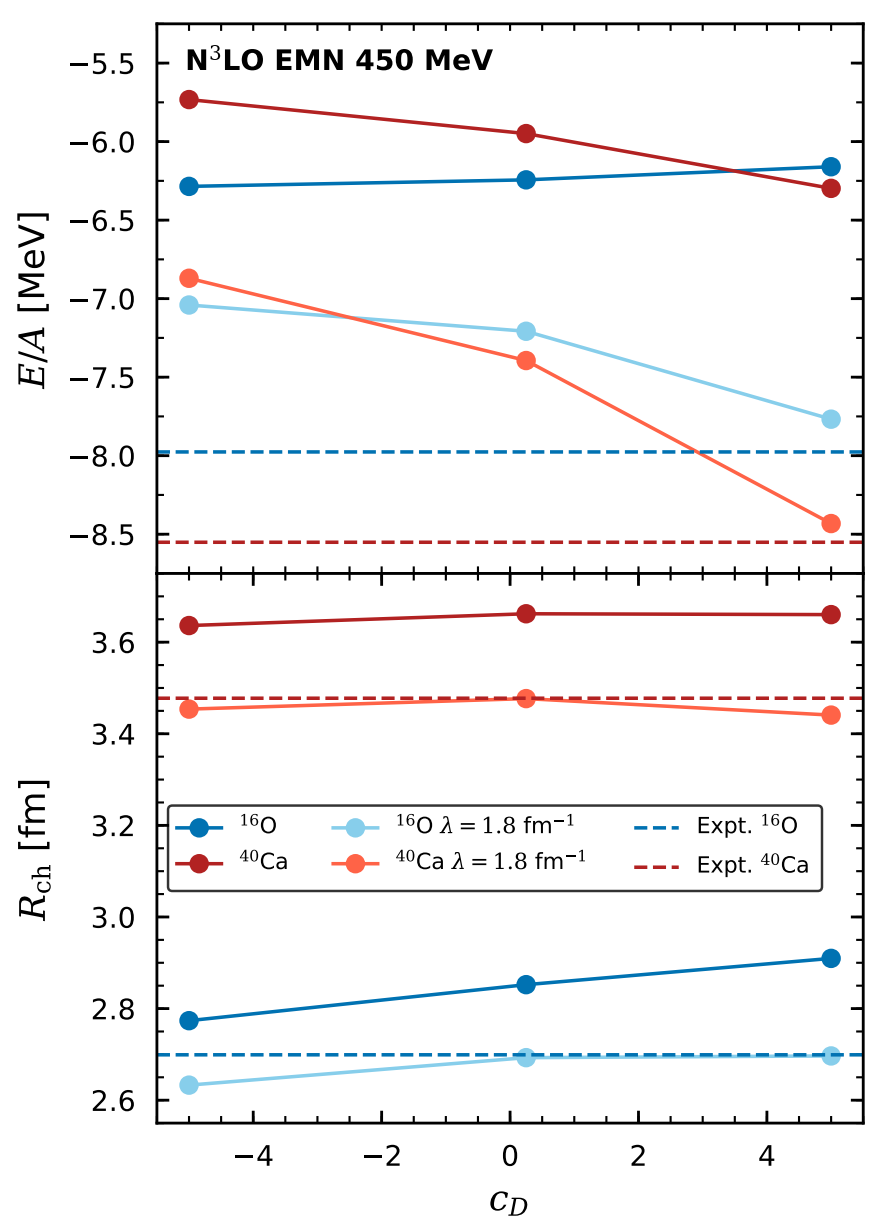

FIG. 8. Ground-state energies (top) and charge radii (bottom panel) for ${ }^{16} \mathrm{O}$ and ${ }^{40} \mathrm{Ca}$ as a function of $c_{D}$ (with corresponding $c_{E}$ value from the triton binding energy, see Table II). Results are shown for unevolved and SRG-evolved potentials with $\lambda=1.8 \mathrm{fm}^{-1}$. The experimental values from Refs. 32, 33] are given by the dashed lines.

mass numbers, and the results for all cutoffs are within the (orange) uncertainty band. The underbinding compared to experiment is expected from the comparison of the $\mathrm{N}^{3} \mathrm{LO}$ EMN $450 \mathrm{MeV}$ interaction with the empirical saturation region (see Fig. 4 in Ref. [6]). The groundstate energies resulting from the $\Delta$-full interaction are in better agreement with experiment, but still underbind the investigated closed-shell nuclei (see also Ref. [1]).

The general trends for the charge radii are similar to the observations for the ground-state energies, with systematic order-by-order convergence, overlapping uncertainty bands, and small $\mathrm{N}^{3} \mathrm{LO}$ cutoff variation. Overall we find too large radii, but the $\mathrm{N}^{2} \mathrm{LO}$ uncertainty band encloses the experimental values, while the $\Delta$-full interaction again exhibits better agreement with experiment. The correlation with the empirical saturation region needs further studies in this case, as one would have expected smaller radii based on nuclear matter saturation for the $\mathrm{N}^{3} \mathrm{LO}$ EMN $450 \mathrm{MeV}$ interaction (see again

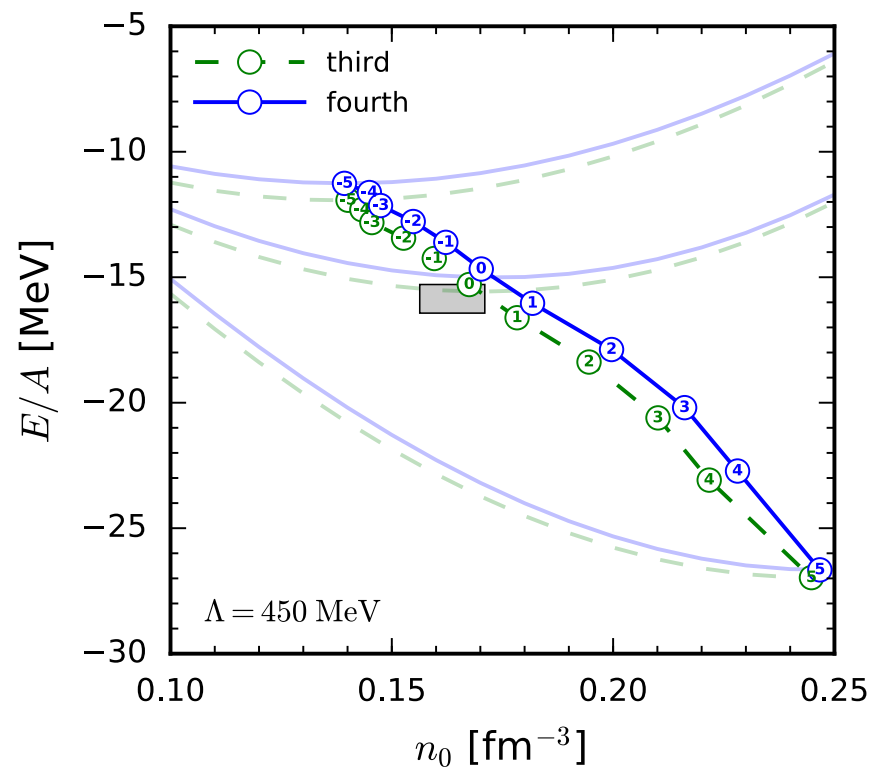

FIG. 9. Same as Fig. 1 but for the $\mathrm{N}^{3} \mathrm{LO}$ EMN $450 \mathrm{MeV}$ interaction. Note the increased range in density and in $c_{D}$ couplings (annotated). We also show the energy per particle for three interactions with $c_{D}=-5,0.25$, and 5 at third and fourth order in MBPT. For $c_{D}=5$, the saturation point is more exploratory, as this is not as constrained in density from our calculations up to densities of $0.25 \mathrm{fm}^{-3}$.

Fig. 4 in Ref. [6]).

Our results for the ground-state energies and charge radii indicate that a realistic description of only the saturation point of nuclear matter may not be sufficient for a realistic description of medium-mass nuclei. To shed more light on this, we study the sensitivity of our results on variations of the $3 N$ couplings $c_{D}$ and $c_{E}$, constrained only by fits to the triton binding energy (see Table I), without the constraint to the empirical saturation region. The results for the ground-state energies and charge radii of ${ }^{16} \mathrm{O}$ and ${ }^{40} \mathrm{Ca}$ as a function of $c_{D}$ are shown in Fig. 8. Although the saturation point of nuclear matter is very sensitive to the values of $c_{D}$, as shown by the large variation in Fig. 9, the variation of the ground-state energy of ${ }^{16} \mathrm{O}$ and ${ }^{40} \mathrm{Ca}$ over the range of $c_{D}=-5 \ldots 5$ is much smaller, which points to lower nuclear matter densities being more relevant, and the variation of the charge radii is very small. We have checked that the weaker impact of $c_{D}$ also persists for the other studied cutoffs at $\mathrm{N}^{3} \mathrm{LO}$. For illustration, Fig. 9 also shows the nuclear matter energy per particle at third and fourth order in MBPT for three $c_{D}$ values $(-5,0.25,5)^{1}$. Tracing these to lower densities around $0.1 \mathrm{fm}^{-3}$ or below gives an energy range for the $c_{D}$ variation, which is much smaller

\footnotetext{
1 Note that the minima of the nuclear matter energy curves can be slightly different from the approximate $c_{D}$ fitting procedure, such that the saturation points may not be exactly the same for the curves and circles in Fig. 9
} 


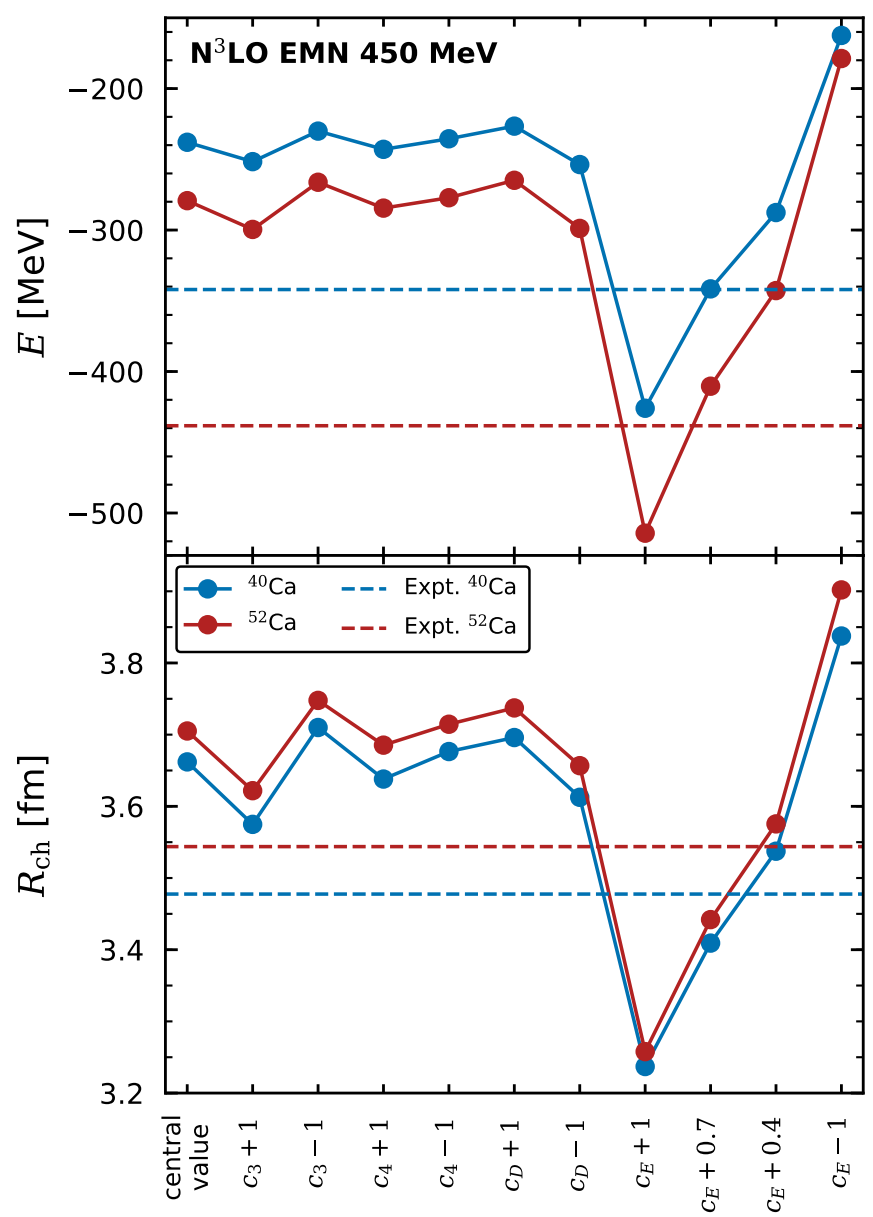

FIG. 10. Ground-state energies (top) and charge radii (bottom panel) for ${ }^{40} \mathrm{Ca}$ and ${ }^{52} \mathrm{Ca}$ for variations of the long-range $3 N$ couplings $c_{3}, c_{4}$ by $\pm 1 \mathrm{GeV}^{-1}$ and the shorter-range $3 N$ couplings $c_{D}, c_{E}$ by \pm 1 . Varying $c_{1}$ by $\pm 1 \mathrm{GeV}^{-1}$ leads to similar results as for $c_{3}$. In addition, we show results for $c_{E}+0.7$ and $c_{E}+0.4$. The first point (central value) is for the original $\mathrm{N}^{3} \mathrm{LO}$ EMN $450 \mathrm{MeV}$ interaction. The experimental values from Refs. 32,34 are given by the dashed lines.

than at saturation density. Even though the change in energy is still larger than for finite nuclei, the lower densities resemble more closely the results for ${ }^{16} \mathrm{O}$ and ${ }^{40} \mathrm{Ca}$. The sensitivity of the ground-state energies in Fig. 8 to $c_{D}$ increases for the SRG-evolved interactions, but is still much smaller than for nuclear matter at saturation density. More work is thus needed to establish in which way nuclear matter properties are most constraining for the development of novel nuclear forces that lead to accurate results for medium-mass and heavy nuclei.

To explore more comprehensively how sensitive our results are to the $3 N$ couplings, we vary their values independently, starting from the $\mathrm{N}^{3} \mathrm{LO}$ EMN $450 \mathrm{MeV}$ interaction. In Fig. 10, we present the ground-state energies and charge radii of the calcium isotopes ${ }^{40} \mathrm{Ca}$ and ${ }^{52} \mathrm{Ca}$ for variations of the long-range $3 N$ couplings $c_{3}, c_{4}$ by $\pm 1 \mathrm{GeV}^{-1}$ and the shorter-range $3 N$ couplings $c_{D}$, $c_{E}$ by \pm 1 . Varying $c_{1}$ by $\pm 1 \mathrm{GeV}^{-1}$ leads to similar

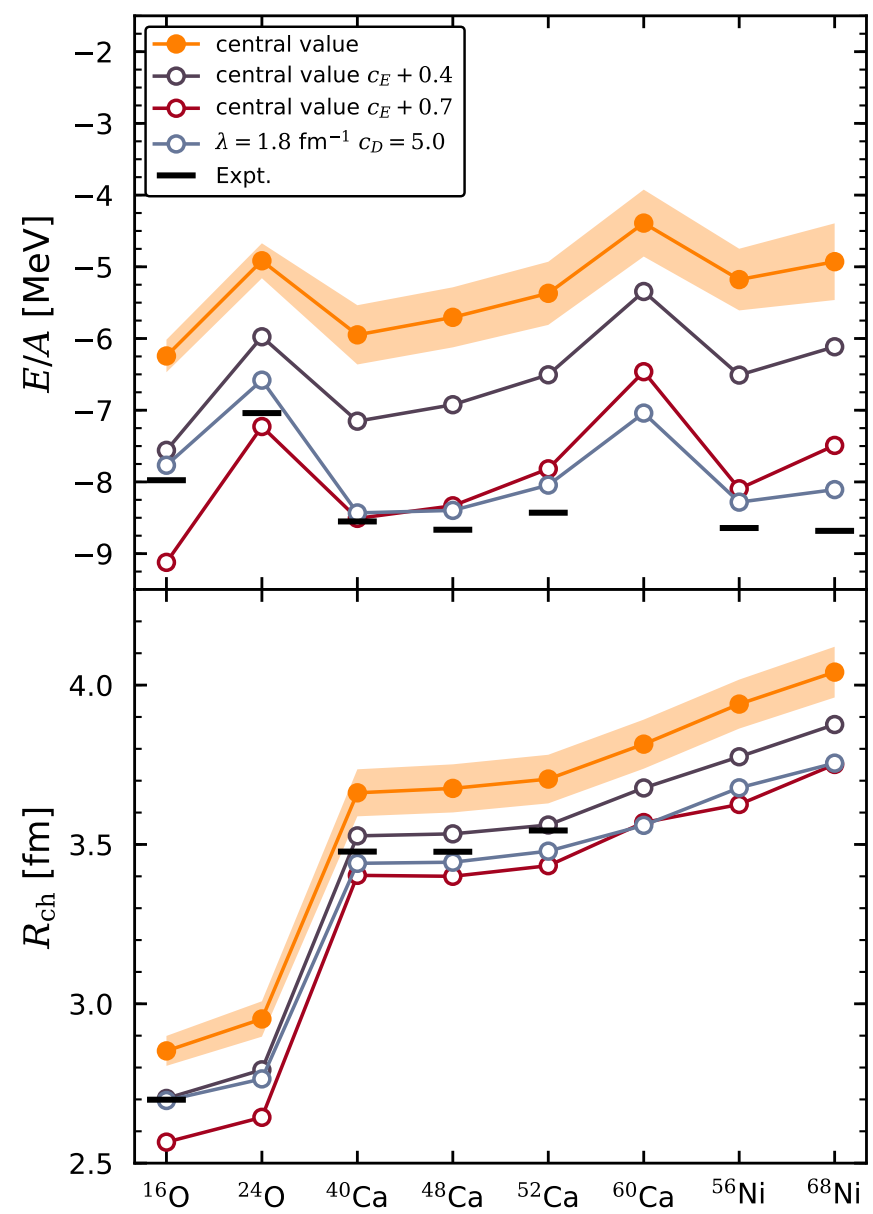

FIG. 11. Ground-state energies per nucleon and charge radii of selected closed-shell oxygen, calcium, and nickel isotopes for the original $\mathrm{N}^{3} \mathrm{LO}$ EMN $450 \mathrm{MeV}$ interaction (central value), the modified $c_{E}+0.4$ as well as $c_{E}+0.7$ interaction, and the SRG-evolved interaction with $\lambda=1.8 \mathrm{fm}^{-1}$ (with $c_{D}=5.0$ ). The orange band represents the $\mathrm{N}^{3} \mathrm{LO}$ uncertainty estimate from Figs. 6 and 7. Experimental values are taken from Refs. 32 34.

results as for $c_{3}$. This exploratory study thus ignores correlations among these low-energy couplings (see, e.g., Ref. [36]). All of our variations have a relatively small impact on energies and radii, apart from variations in $c_{E}$, which lead to significant changes of $\Delta E \approx 260 \mathrm{MeV}$ and $\Delta R_{\mathrm{ch}} \approx 0.6 \mathrm{fm}$ for ${ }^{40} \mathrm{Ca}$ and similarly for ${ }^{52} \mathrm{Ca}$. We therefore consider two additional variations, $c_{E}+0.4$ and $c_{E}+0.7$. The latter reproduces well the ground-state energy of ${ }^{40} \mathrm{Ca}$ and leads to an improvement for ${ }^{52} \mathrm{Ca}$. We also found that setting all $3 N$ couplings but the $c_{3}$ contribution to zero leads to similar results as the original $\mathrm{N}^{3} \mathrm{LO}$ EMN $450 \mathrm{MeV}$ interaction.

In Fig. 11 we show results for selected closed-shell nuclei exploring some of these variations compared to the original $\mathrm{N}^{3} \mathrm{LO}$ EMN $450 \mathrm{MeV}$ interaction (labeled central value). The interaction $c_{E}+0.7$ leads to increased binding and a better description of ground-state energies, whereas $c_{E}+0.4$ leads to improved agreement with exper- 
TABLE II. ${ }^{3} \mathrm{H}$ ground-state energy and charge radius for the $\mathrm{N}^{3} \mathrm{LO}$ EMN $450 \mathrm{MeV}$ interaction with modified $3 N$ couplings.

\begin{tabular}{lccccc}
\hline \hline & Expt. & $c_{E}+0.4$ & $c_{E}+0.7$ & $c_{3}+1$ & $c_{4}+1$ \\
\hline$E[\mathrm{MeV}]$ & -8.48 & -9.81 & -11.18 & -8.40 & -8.74 \\
$R_{\text {ch }}[\mathrm{fm}]$ & 1.575 & 1.469 & 1.381 & 1.576 & 1.550 \\
\hline \hline
\end{tabular}

imental charge radii. However, despite these promising results for medium-mass nuclei, the modifications of the $3 N$ couplings lead to a heavily overbound ${ }^{3} \mathrm{H}$ with a far too small charge radius, see Table II This shows the difficulty in achieving a realistic simultaneous description of few-body systems, medium-mass nuclei, and nuclear matter just by varying the $3 N$ couplings based on this set of $N N$ interactions, and also emphasizes the need for reliable theoretical uncertainties. In addition, we show in Fig. 11 results for the SRG-evolved interaction with $\lambda=1.8 \mathrm{fm}^{-1}$ (with $c_{D}=5.0$ ), for which we find groundstate energies and charge radii of ${ }^{16} \mathrm{O}$ and ${ }^{40} \mathrm{Ca}$ in good agreement with experiment (see Fig. 8). Figure 11 shows that this interaction is also able to reproduce energies and radii of other closed-shell nuclei in good agreement with experiment (considering the $\mathrm{N}^{3} \mathrm{LO}$ uncertainties). By construction, it still reproduces the experimental triton binding energy.

\section{SUMMARY AND OUTLOOK}

Our work presents the first $a b$ initio calculations of medium-mass nuclei with $N N+3 N$ interactions to $\mathrm{N}^{3} \mathrm{LO}$. We have studied in detail ground-state energies and charge radii of closed-shell nuclei up to nickel for unevolved as well as momentum-space SRG-evolved $N N+3 N$ interactions with reasonable saturation properties. In addition, we have explored a consistent momentum-space SRG evolution of these $N N+3 N$ interactions. In general, the ground-state energies predicted by the employed interactions significantly underbind all oxygen, calcium, and nickel isotopes studied here and lead to too large charge radii. Remarkably, the results exhibit only a weak dependence on the cutoff scale. The uncertainty estimates at $\mathrm{N}^{2} \mathrm{LO}$ and $\mathrm{N}^{3} \mathrm{LO}$ for the order-byorder convergence of the chiral expansion show a systematic behavior with the $\mathrm{N}^{2} \mathrm{LO}$ band enclosing the $\mathrm{N}^{3} \mathrm{LO}$ band for all studied nuclei. For comparison, we also employed the $\Delta$-full interaction of Ref. [11] at $\mathrm{N}^{2} \mathrm{LO}$, which gives improved agreement with experimental binding energies and charge radii.

While underbinding was expected from the saturation point of the corresponding interactions, the behavior of the charge radii and their correlation with the saturation point did not systematically follow nuclear matter. For a more detailed study of this correlation, we varied the $3 N$ couplings under the constraint that the ${ }^{3} \mathrm{H}$ binding energy agrees with experiment, and studied the resulting sensitivity of observables in both systems. While the ground-state energies of ${ }^{16} \mathrm{O}$ and ${ }^{40} \mathrm{Ca}$ changed by $<1 \mathrm{MeV}$ for unevolved interactions, the change in saturation energy was $15 \mathrm{MeV}$ over the studied $c_{D}$ range. This indicated that nuclear matter physics at lower densities is also relevant. When the ${ }^{3} \mathrm{H}$ constraint was relaxed, we found the largest impact on energies and radii from changes of the short-range $3 N$ coupling. However, while these variations allowed us to construct $N N+3 N$ interactions that lead to an improved description of mediummass nuclei, the resulting nuclear forces substantially overbind the triton and underestimate its charge radius. These findings show that the connection between light nuclei, medium-mass nuclei, and nuclear matter is quite intricate and requires further investigations. An improved understanding of this is especially relevant for the derivation and construction of accurate next-generation $N N$ and many-body interactions in chiral EFT.

\section{ACKNOWLEDGMENTS}

We thank K. Vobig for useful discussions. We also thank R. Machleidt and A. Ekström for providing us with the EMN and $\Delta$-full potentials, respectively, as well as S. R. Stroberg for discussions on the IM-SRG code 37 . This work was supported by the Deutsche Forschungsgemeinschaft (DFG, German Research Foundation) Projektnummer 279384907 - SFB 1245, the BMBF under Contract No. 05P18RDFN1, the US Department of Energy, the Office of Science, the Office of Nuclear Physics, and SciDAC under awards DE_SC00046548 and DE_AC02_05CH11231, and by the Cluster of Excellence "Precision Physics, Fundamental Interactions, and Structure of Matter" (PRISMA+ EXC 2118/1) funded by DFG within the German Excellence Strategy (Project ID 39083149). C.D. acknowledges support by the Alexander von Humboldt Foundation's Feodor-Lynen Fellowship program. Computational resources have been provided by the Lichtenberg high performance computer of the TU Darmstadt.
[1] A. Ekström, G. R. Jansen, K. A. Wendt, G. Hagen, T. Papenbrock, B. D. Carlsson, C. Forssén, M. HjorthJensen, P. Navrátil, and W. Nazarewicz, Phys. Rev. C 91, 051301(R) (2015).
[2] B. D. Carlsson, A. Ekström, C. Forssén, D. F. Strömberg, G. R. Jansen, O. Lilja, M. Lindby, B. A. Mattsson, and K. A. Wendt, Phys. Rev. X 6, 011019 (2016).

[3] J. E. Lynn, I. Tews, J. Carlson, S. Gandolfi, A. Gezerlis, 
K. E. Schmidt, and A. Schwenk, Phys. Rev. Lett. 116, 062501 (2016).

[4] P. Reinert, H. Krebs, and E. Epelbaum, Eur. Phys. J. A 54, 86 (2018).

[5] D. R. Entem, R. Machleidt, and Y. Nosyk, Phys. Rev. C 96, 024004 (2017).

[6] C. Drischler, K. Hebeler, and A. Schwenk, Phys. Rev. Lett. 122, 042501 (2019).

[7] K. Hebeler, S. K. Bogner, R. J. Furnstahl, A. Nogga, and A. Schwenk, Phys. Rev. C 83, 031301(R) (2011).

[8] J. Simonis, K. Hebeler, J. D. Holt, J. Menéndez, and A. Schwenk, Phys. Rev. C 93, 011302(R) (2016).

[9] G. Hagen, A. Ekström, C. Forssén, G. R. Jansen, W. Nazarewicz, T. Papenbrock, K. A. Wendt, S. Bacca, N. Barnea, B. Carlsson, C. Drischler, K. Hebeler, M. Hjorth-Jensen, M. Miorelli, G. Orlandini, A. Schwenk, and J. Simonis, Nat. Phys. 12, 186 (2016).

[10] J. Simonis, S. R. Stroberg, K. Hebeler, J. D. Holt, and A. Schwenk, Phys. Rev. C 96, 014303 (2017).

[11] A. Ekström, G. Hagen, T. D. Morris, T. Papenbrock, and P. D. Schwartz, Phys. Rev. C 97, 024332 (2018).

[12] S. Binder, J. Langhammer, A. Calci, and R. Roth, Phys. Lett. B 736, 119 (2014).

[13] A. Tichai, J. Langhammer, S. Binder, and R. Roth, Phys. Lett. B 756, 283 (2016).

[14] T. D. Morris, J. Simonis, S. R. Stroberg, C. Stumpf, G. Hagen, J. D. Holt, G. R. Jansen, T. Papenbrock, R. Roth, and A. Schwenk, Phys. Rev. Lett. 120, 152503 (2018).

[15] R. Machleidt, private communication, (2017-2018).

[16] E. Epelbaum, A. Nogga, W. Glöckle, H. Kamada, U.G. Meißner, and H. Witała, Phys. Rev. C 66, 064001 (2002).

[17] K. Hebeler, H. Krebs, E. Epelbaum, J. Golak, and R. Skibinski, Phys. Rev. C 91, 044001 (2015).

[18] K. Hebeler, Phys. Rev. C 85, 021002(R) (2012).

[19] K. Tsukiyama, S. K. Bogner, and A. Schwenk, Phys. Rev. Lett. 106, 222502 (2011).

[20] H. Hergert, S. K. Bogner, S. Binder, A. Calci, J. Langhammer, R. Roth, and A. Schwenk, Phys. Rev. C 87,
034307 (2013)

[21] H. Hergert, S. K. Bogner, T. D. Morris, A. Schwenk, and K. Tsukiyama, Phys. Rept. 621, 165 (2016).

[22] S. R. Stroberg, A. Calci, H. Hergert, J. D. Holt, S. K. Bogner, R. Roth, and A. Schwenk, Phys. Rev. Lett. 118, 032502 (2017).

[23] S. K. Bogner, R. J. Furnstahl, and R. J. Perry, Phys. Rev. C 75, 061001(R) (2007).

[24] S. K. Bogner, R. J. Furnstahl, and A. Schwenk, Prog. Part. Nucl. Phys. 65, 94 (2010).

[25] S. R. White, J. Chem. Phys. 117, 7472 (2002).

[26] W. Magnus, Commun. Pure Appl. Math. 7, 649 (1954).

[27] T. D. Morris, N. M. Parzuchowski, and S. K. Bogner, Phys. Rev. C 92, 034331 (2015).

[28] C. Patrignani et al. (Particle Data Group), Chin. Phys. C 40, 100001 (2016).

[29] J. L. Friar, J. Martorell, and D. W. L. Sprung, Phys. Rev. A 56, 4579 (1997).

[30] L. L. Foldy and S. A. Wouthuysen, Phys. Rev. 78, 29 (1950).

[31] A. Ong, J. C. Berengut, and V. V. Flambaum, Phys. Rev. C 82, 014320 (2010).

[32] M. Wang, G. Audi, F. G. Kondev, W. Huang, S. Naimi, and X. Xu, Chin. Phys. C 41, 030003 (2017).

[33] I. Angeli and K. Marinova, Atomic Data and Nuclear Data Tables 99, 69 (2013).

[34] R. F. Garcia Ruiz, M. L. Bissell, K. Blaum, A. Ekström, N. Frömmgen, G. Hagen, M. Hammen, K. Hebeler, J. D. Holt, G. R. Jansen, M. Kowalska, K. Kreim, W. Nazarewicz, R. Neugart, G. Neyens, W. Nörtershäuser, T. Papenbrock, J. Papuga, A. Schwenk, J. Simonis, K. A. Wendt, and D. T. Yordanov, Nat. Phys. 12, 594 (2016).

[35] E. Epelbaum, H. Krebs, and U.-G. Meißner, Eur. Phys. J. A 51, 53 (2015).

[36] M. Hoferichter, J. Ruiz de Elvira, B. Kubis, and U.-G. Meißner, Phys. Rev. Lett. 115, 192301 (2015).

[37] S. R. Stroberg, https://github.com/ragnarstroberg/imsrg (2017). 